\section{Malignant melanoma metastasis to the colon}

The large intestine is the least common site in the gastrointestinal tract for malignant melanoma metastases, and does not appear to be a site of primary tumor [1]. Endoscopically, metastatic melanoma may present as submucosal nodules, polypoid masses, or colonic intussusception [1, $2]$, or as a large exophytic mass that mimicks colon cancer [3]. The liver is the site most commonly affected by melanoma metastases, and the most common cause of death is respiratory failure resulting from pulmonary invasion by tumor cells [2]. We report here a case which demonstrates the typical findings of metastatic melanoma involving the colon.

An 84-year-old woman presented at our institution with a 3-week history of progressive dyspnea and cough. Multiple nodular opacities were noted bilaterally on an initial chest radiograph. There were no cutaneous lesions or peripheral lymphadenopathy found on physical examination. Her laboratory data were within reference ranges, except for the hematocrit, which was $35.8 \%$ (normal range $37 \%-47 \%$ ) and the carbohydrate-associated antigen 125 (CA125), which was $92.61 \mathrm{U} / \mathrm{ml}$ (normal range $<35 \mathrm{U} / \mathrm{ml}$ ). Contrast-enhanced computed tomography showed multiple nodular densities over both lung fields and multiple low-density masses within the liver parenchyma.

Colonoscopy revealed a polypoid lesion, $12 \mathrm{~mm}$ in diameter, in the sigmoid colon (Figure 1a). The lesion was black-pigmented, with a central ulcer and a darkly pigmented margin. Multiple volcano-like mucosal elevations, 2-4 mm in diameter, were found in the sigmoid colon and rectum (Figure $\mathbf{1 b}, \mathbf{c}$ ). Histological assessment of the biopsy specimens showed a picture of malignant melanoma (Figure 2a); immunohistochemical staining revealed positivity for S100 (Figure $\mathbf{2 b}$ ) and HMB-45 (Figure $2 \mathbf{c}$ ). A diagnosis of metastatic melanoma was made. The pulmonary and hepatic lesions were considered to be metastatic tumors. No clinically apparent primary focus was found. The
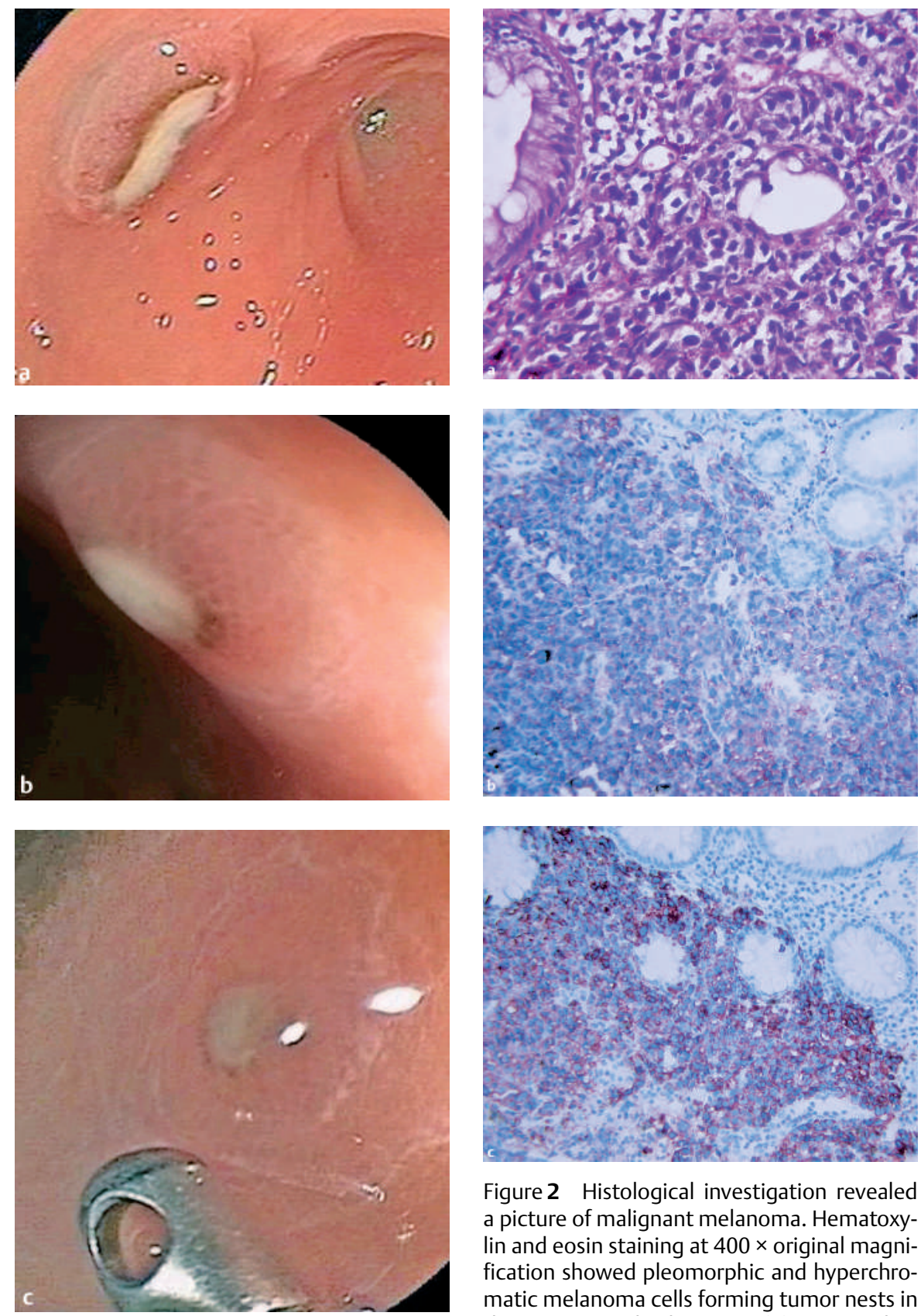

Figure 2 Histological investigation revealed a picture of malignant melanoma. Hematoxylin and eosin staining at $400 \times$ original magnification showed pleomorphic and hyperchromatic melanoma cells forming tumor nests in the mucosa and submucosa (a). Immunohistochemical staining revealed positivity for S100 (b) and for HMB-45 (c) $(200 \times$ original magnification). ed margin (a). There were volcano-like mucosal elevations around the polypoid lesion, with ulcerated tops and darkly pigmented margins (b,c).

patient died of respiratory failure 9 days later. 


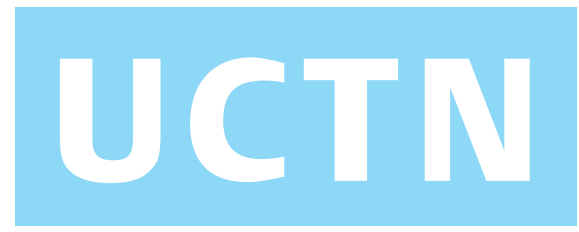

S.-L. Yan, C.-C. Yang, Y.-T. Liu Division of Gastroenterology, Department of Internal Medicine, Show-Chwan Memorial Hospital, Changhua, Taiwan.
References

${ }^{1}$ Blecker D, Abraham S, Furth EE, Kochman ML. Melanoma in the gastrointestinal tract. Am J Gastroenterol 1999; 94: 3427-3433

2 Lain EL, Orengo IF, Rosen T. Metastatic melanoma. J Am Acad Dermatol 2005; 53: $314-$ 317

${ }^{3}$ Eloubeidi MA, Gaede JT, Davis WZ. Isolated metastatic melanoma to the colon mimicking colon cancer. Gastrointest Endosc 2000; 52: $751-752$
Corresponding author

\section{Y.-T. Liu, M.D.}

Division of Gastroenterology Department of Internal Medicine Show-Chwan Memorial Hospital No. 542, Sec 1, Chung-Shang Rd Changhua 500

Taiwan

Fax: $\quad+886-4-7233190$

E-mail: yslcsmu@yahoo.com 